Pacific Journal of Mathematics

AN ALGEBRAIC EXTENSION OF THE LAX-MILGRAM 


\title{
AN ALGEBRAIC EXTENSION OF THE LAX-MILGRAM THEOREM
}

\author{
GABRIEL M. M. OBI
}

In this work a Lax-Milgram type theorem is proved for quadratic spaces over a division ring $K$ with involution *, say, whose center contains an ordered domain $P$ such that for every element $a$ in $K, a a^{*}=|a|^{2}$, (where $|a|$, the absolute value of $a$, is in $P^{+}$which is the set of positive elements of $P$ ), and for every element $b$ in $P^{+}$there exists an element $c$ in $P^{+}$, denoted by $b^{1 / 2}$, such that $c^{2}=b$. Specifically, with the above assumptions on $K$, the following is proved:

Let $\left(H_{i}, \Phi_{i}\right) i=1,2$ be quadratic spaces over $K$ such that for each $u$ in $H_{2} \sup \left|\Phi_{2}(u, v)\right|\left(\left|\Phi_{2}(v, v)\right|^{1 / 2}\right)^{-1}$ exists and equals $\left|\Phi_{2}(u, u)\right|^{1 / 2}$. Let $B: H_{1} \times H_{2} \rightarrow K$ be an orthocontinuous bilinear form satisfying:

(i ) $\inf _{x \neq 0} \sup _{y \neq 0}|B(x, y)|\left(\left|\Phi_{1}(x, x)\right|^{1 / 2}\left|\Phi_{2}(y, y)\right|^{1 / 2}\right)^{-1}=\gamma$ exists and $\gamma-\delta$ is in $P^{+}$for some $\delta$ in $P^{+}$.

(ii) $\sup |B(x, y)|$ exists and is in $P^{+}$for all $y \neq 0 x \in H$.

Then given any orthocontinuous linear functional $\phi$ on $H_{2}$ whose kernel is splitting there exists a unique element $x_{0}$ in $H_{1}$ such that $\phi(y)=B\left(x_{0}, y\right)$ for all $y$ in $H_{2}$.

Moreover

$$
\delta^{-1} \sup _{y \neq 0}|\phi(y)|\left(\left|\Phi_{2}(y, y)\right|^{1 / 2}\right)^{-1}-\left|\Phi_{1}\left(x_{0}, x_{0}\right)\right|^{1 / 2} \in P^{+} \cup\{0\} .
$$

1. Introduction. Motivated by what he referred to as "happy accidents in the Hilbert space theory that correlate algebraic and topological considerations" Piziak [3] proposed an algebraic approach to the study of sesquilinear forms in infinite dimensions. In this approach he introduced the notion of quadratic spaces by the means of which he obtained an algebraic generalization of some Hilbert space results. He proved an algebraic version of the Riesz-Frechet Representation Theorem and discussed continuity all in the algebraic context of a vector space over a division ring in which no natural topology is present.

We here consider an algebraic extension of the Lax-Milgram Theorem, a variant of the Riesz Representation Theorem.

Our results are of pure algebra. We have not assumed a topology either on the division ring or on the vector space which we considered. It is thus interesting to note that these results imply their analogous standard topological results in the context of Hilbert space. 
2. Preliminaries.

Definition 2.1. [2], [3]. A quadratic space is a triple $(K, H, \Phi)$ where $K$ is a division ring with involution, ${ }^{*}, H$ is a left vector space over $K$, and $\Phi$ is a nondegenerate orthosymmetric *-sesquilinear form on $H$ with respect to the involutive anti-automorphism * of $K$.

Where there is no confusion we shall denote a quadratic space simply by $H$ or $(H, \Phi)$.

Definition 2.2. Let $H$ be a quadratic space. For $x, y$ in $H$ we say $x$ is orthogonal to $y$ and write $x \perp y$ if $\Phi(x, y)=0$.

We note that since $\Phi$ is orthosymmetric $x \perp y$ implies $y \perp x$ and vice-versa.

Notation 2.3. For any subset $M$ of $H$, put

$$
M^{\perp}=\{y \text { in } H: \Phi(x, y)=0 \text { for all } x \text { in } M\} .
$$

It is easy to see that $M^{\perp}$ is a subspace of $H$ for every subset $M$ of $H$. A vector $x$ in $H$ is said to be isotropic if $x \perp x$ and anisotropic otherwise. If every nonzero vector in $H$ is anisotropic then $H$ is called an anisotropic quadratic space.

Definition 2.4. We call a subspace $M$ of $H \perp$-closed iff $M=M^{\perp \perp}$. We say $M$ splits $H$ if $H=M \oplus M^{\perp}$ and we say $M$ is semi-simple if $M \cap M^{\perp}=\{0\}$.

3. Lax-Milgram theorem. The Lax-Milgram states, [4]:

Let $H$ be a Hilbert space (real or complex) and $B$ a bilinear form on $H$ such that

(i) $|B(x, y)| \leqq \rho\|x\|\|y\|$ for all $x, y$ in $H$ and some positive real constant $\rho$.

(ii) There exists a positive real number $\delta$ such that $B(x, x)>$ $\delta\|x\|^{2}$ for every $x$ in $H$.

Then there exists a unique bounded linear operator $T$ on $H$ such that

(a) $\langle x, y\rangle=B(x, T y)$ for all $x, y$ in $H$.

(b) $\|T\| \leqq \delta^{-1}$.

(Here $\langle\cdot, \cdot\rangle$ denotes the inner product on $H$.)

An observation of this theorem shows that condition (i) implies that $B$ is continuous and (ii) implies that

(ii) (a) $B$ is positive definite.

(ii) (b) The maps $B_{x}(\cdot), B^{y}(\cdot): H \rightarrow C$ defined by $B_{x}(y)=B(x, y)$; 
$B^{y}(x)=B(x, y)$ are nontrivial for each $x$ (resp. each $y$ ) in $H$ i.e., $B^{y}(x)=0$ for all $x$ in $H$ implies $y=0$ and $B_{x}(y)=0$ for all $y$ in $H$ implies $x=0$.

Also the conclusion of the theorem can be reframed thus:

Then given any bounded linear functional $f$ on $H$ there exists a unique vector $u_{0}$ in $H$ such that $f(u)=B\left(u_{0}, u\right)$ for all $u$ in $H$ and $\left\|u_{0}\right\| \leqq \delta^{-1}\|f\|$.

We shall now generalize this result to quadratic spaces.

Definition 3.1. Let $\left(H_{i}, \Phi_{i}\right), i=1,2$ be quadratic spaces. Let $L: H_{1} \rightarrow H_{2}$ be a linear transformation such that $L\left(M^{\perp \perp}\right) \cong L(M)^{\perp \perp}$ for every subspace $M$ of $H_{1}$. Then $L$ is said to be orthocontinuous.

Proposition 3.2. [2], [3]. Let $\left(H_{i}, \Phi_{i}\right), i=1,2$ be quadratic spaces and $L: H_{1} \rightarrow H_{2}$ be a linear transformation. Then the following are equivalent:

(i ) $M=M^{\perp \perp}$ implies $L^{-1}(M)=L^{-1}(M)^{\perp \perp}$ for all subspaces $M$ of $\mathrm{H}_{2}$.

(ii) If $M$ is a $\perp$-closed subspace of $H_{2}$ then $L^{-1}(M)$ is a 1 closed subspace of $H_{1}$.

(iii) $L\left(M^{\perp \perp}\right) \subseteq L(M)^{\perp \perp}$ for all subspaces $M$ of $H_{1}$.

(iv) $L^{-1}(M)^{\perp \perp} \subseteq L^{-1}\left(M^{\perp \perp}\right)$ for all subspaces $M$ of $H_{2}$.

(v) $L$ is orthocontinuous.

Definition 3.3. [2], [3]. Let $(K, H, \Phi)$ be a quadratic space. A linear map $\dot{\phi}: H \rightarrow K$ is called an orthocontinuous linear functional if $(\operatorname{Ker} \phi)^{\perp \perp}=\operatorname{Ker} \phi$.

Now, in Hilbert space a bilinear form $B(x, y)$ is continuous iff there exists a continuous linear transformation $L$ on $H$ such that $B(x, y)=\langle L x, y\rangle$ for all $x, y$ in $H$. Motivated by this fact we make the following definition:

Definition 3.4. Let $\left(H_{i}, \Phi_{i}\right), i=1,2$ be quadratic spaces. A bilinear form $B: H_{1} \times H_{2} \rightarrow K$ is said to be orthocontinuous if there exists an orthocontinuous linear operator $L: H_{1} \rightarrow H_{2}$ such that $B(x, y)=\Phi_{2}(L x, y)$ for every $(x, y)$ in $H_{1} \times H_{2}$.

Proposition 3.5. Let $\left(H_{i}, \Phi_{i}\right), i=1,2$ be quadratic space and $B: H_{1} \times H_{2} \rightarrow K$ be an orthocontinuous bilinear form. Then the mappings $B_{x}(\cdot): H_{2} \rightarrow K, B^{y}(\cdot): H_{1} \rightarrow K$ are orthocontinuous.

Proof. Let $L$ be as in 3.4 .

$\operatorname{Ker} B_{x}(\cdot)=\{y: B(x, y)=0\}$ 


$$
\begin{aligned}
& =\left\{y: \Phi_{2}(L x, y)=0\right\} \\
& =(L x)^{\perp} \text { a } \perp \text {-closed subspace }
\end{aligned}
$$

Also

$$
\begin{aligned}
\operatorname{Ker} B^{y}(\cdot)= & \{x: B(x, y)=0\} \\
= & \left\{x: \Phi_{2}(L x, y)=0\right\} \\
= & \left\{x: L x \in y^{\perp}\right\} \\
= & L^{-1}\left(y^{\perp}\right) \text { a } \perp \text {-closed subspace } \\
& \text { since } L \text { is orthocontinuous. }
\end{aligned}
$$

We note that the orthocontinuity of $B$ implies that of $B_{x}$ and $B^{y}$. The converse is not true in general, however. This is a consequence of [3, Theorem 3].

Proposition 3.6. Let $\left(H_{i}, \Phi_{i}\right), i=1,2$ be quadratic spaces. Let $M=\{0\} \times H_{2} \cong H_{2}$ and $\pi: H_{1} \times H_{2} \rightarrow H_{2}$ be the projection $\pi((x, y))=y$ for all $(x, y)$ in $H_{1} \times H_{2}$. Then $\pi$ maps $\perp$-closed subspaces of $H_{1} \times H_{2}$ onto $\perp$-closed subspaces of $H_{2}$.

Proof. We first note that any subspace of $H_{1} \times H_{2}$ of the form $A \times B$ where $A$ is a subspace of $H_{1}$ and $B$ a subspace of $H_{2}$ is $\perp$ closed in $H_{1} \times H_{2}$ iff $A$ is $\perp$-closed in $H_{1}$ and $B$ is $\perp$-closed in $H_{2}$. Hence a subspace $B$ of $H_{2}$ is $\perp$-closed iff $\pi^{-1}(B)$ is $\perp$-closed. We also note that $\left(H_{1} \times H_{2}, \Phi_{1} \oplus \Phi_{2}\right)$ is a quadratic space [2] and that $M$ is a semi-simple splitting subspace. Let $\Phi_{M}$ be the restriction of $\Phi_{1} \oplus \Phi_{2}$ to $M$. Then $\left(M, \Phi_{M}\right)$ is a quadratic space. Since $\pi$ is a homomorphism there exists an isomorphism $\phi: H_{1} \times H_{2} / H_{1} \times\{0\} \rightarrow M$. For $u, v$ in $H_{1} \times H_{2} / H_{1} \times\{0\}$ define

$$
\Psi(u, v)=\Phi_{M}(\phi(u), \phi(v)) .
$$

Then since $\phi$ is $1-1$ onto we have that $\Psi$ is a nondegenerate orthosymmetric and *-sesquilinear form on $H_{1} \times H_{2} / H_{1} \times\{0\}$. Let $A \subseteq$ $H_{1} \times H_{2} / H_{1} \times\{0\}$. If $u$ is in $A^{\perp}$ then

$$
\begin{aligned}
& \Phi_{M}(\phi(u), \phi(v))=0 \text { for all } v \text { in } A \\
& \Longleftrightarrow \phi(u) \in \phi(A)^{\perp} .
\end{aligned}
$$

Thus $\phi\left(A^{\perp}\right)=\phi(A)^{\perp}$. Also if $B \leqq M$ then there exists $A \leqq H_{1} \times$ $H_{2} / H_{1} \times\{0\}$ such that $\phi(A)=B$. Suppose $B=B^{\perp \perp}$ then

$$
\begin{aligned}
A & =\phi^{-1}(B) \\
& =\phi^{-1}\left(B^{\perp \perp}\right) \\
& =\phi^{-1}\left(\phi(A)^{\perp \perp}\right)
\end{aligned}
$$




$$
\begin{aligned}
& =\phi^{-1}\left(\phi\left(A^{\perp \perp}\right)\right) \text { by the above argument } \\
& =A^{\perp \perp} .
\end{aligned}
$$

Hence $\phi, \phi^{-1}$ map $\perp$-closed subspaces onto $\perp$-closed subspaces. Now consider the following diagram where $p$ is the canonical projection.

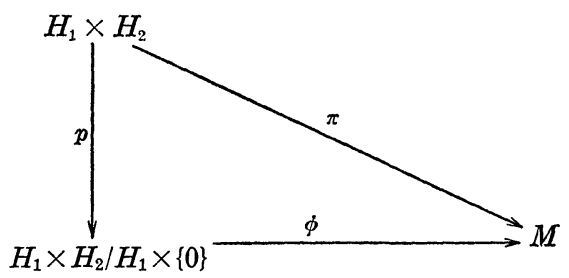

A subspace $B$ of $H_{1} \times H_{2} / H_{1} \times\{0\}$ is $\perp$-closed iff $p^{-1}(B)$ is $\perp$-closed. Indeed let $B$ be $\perp$-closed then $\phi(B)$ is $\perp$-closed and $\pi^{-1}(\phi(B))=p^{-1}(B)$ is $\perp$-closed since $\pi$ being a projection is orthocontinuous. Also if $p^{-1}(B)$ is $\perp$-closed then since $p^{-1}(B)=\pi^{-1}(\phi(B))$ we must have that $\phi(B)$ is $\perp$-closed by the observation at the beginning of the proof. Hence $B=\phi^{-1}(\phi(B))$ is $\perp$-closed. Finally if $A$ is $\perp$-closed in $H_{1} \times H_{2}$, let $B$ be such that $p^{-1}(B)=A$. Then

$$
\begin{aligned}
\pi(A) & =\phi(p(A)) \\
& =\phi\left(p\left(p^{-1}(B)\right)\right) \\
& =\phi(B)
\end{aligned}
$$

which is $\perp$-closed.

LEMMA 3.7. Let $B$ be an orthocontinuous bilinear form on $H_{1} \times H_{2}$. Let $L$ be such that $B(x, y)=\Phi_{2}(L x, y)$ by Definition 3.4. If $B_{x}(\cdot), B^{y}(\cdot)$ are nontrivial for each $x$ in $H_{1}$ and $y$ in $H_{2}$, then $L$ is 1-1 and onto.

Proof. Suppose $L x_{1}=L x_{2}$. Then

$$
\Phi_{2}\left(L x_{1}-L x_{2}, v\right)=0 \text { for all } v \text { in } H_{2},
$$

therefore

$$
\begin{aligned}
B\left(x_{1}-x_{2}, v\right) & =\Phi_{2}\left(L\left(x_{1}-x_{2}\right), v\right) \\
& =\Phi_{2}\left(L x_{1}-L x_{2}, v\right) \\
& =0
\end{aligned}
$$

for all $v$ in $H_{2}$. Since $B_{x}(\cdot)$ is nontrivial we must have that $x_{1}-x_{2}=0$ or $x_{1}=x_{2}$. Thus $L$ is $1-1$. To show that $L$ is onto we note that since $L$ is orthocontinuous its graph, $G(L)$, is a $\perp$-closed 
subspace of $\left(H_{1} \times H_{2}, \Phi_{1} \oplus \Phi_{2}\right)$. Hence $\pi(G(L))$ is $\perp$-closed in $H_{2}$. Suppose $L\left(H_{1}\right)=\pi(G(L)) \neq H_{2}$. Then there exists a nonzero vector $x$ in $H_{2}$ such that for all $x$ in $H_{1}, \Phi_{2}(L x, z)=0$ [4]. Thus

$$
\begin{aligned}
& 0=\Phi_{2}(L x, z) \\
& \quad=B(x, z) \\
& B^{z}(x)
\end{aligned}
$$

for every $x$ in $H_{1}$. Since $B^{y}(\cdot)$ is nontrivial we must have $z=0$. This contradiction establishes the result.

LEMMA 3.8. Let $S: H_{1} \rightarrow H_{2}$ be an orthocontinuous linear transformation from a quadratic space $H_{1}$ to a quadratic space $H_{2}$. If $S^{-1}$ exists then $\left(S^{*}\right)^{-1}$ exists and $\left(S^{*}\right)^{-1}=\left(S^{-1}\right)^{*}$.

Proof. Since $\operatorname{Im}\left(S^{*}\right)=\operatorname{Ker}(S)^{\perp}$ and $\operatorname{Im}(S)=\operatorname{Ker}\left(S^{*}\right)^{\perp}$ [3], and since $S$ is $1-1$ onto because of the existence of $S^{-1}$, we must have that $S^{*}$ is $1-1$ and onto and hence that $\left(S^{*}\right)^{-1}$ exists. Also since $S$ is orthocontinuous the graph of $S^{-1}$ is $\perp$-closed in $\left(H_{2} \times H_{1}, \Phi_{2} \oplus \Phi_{1}\right)$. So $S^{-1}$ is a $C D D$ transformation [2]. Let $x$ be in the domain of $S^{-1}$ and $y$ in the domain of $\left(S^{*}\right)^{-1}$. Then since $S$ is $C D D$ we have that

$$
\begin{aligned}
\Phi_{2}\left(x,\left(S^{*}\right)^{-1} y\right) & =\Phi_{2}\left(S u,\left(S^{*}\right)^{-1} y\right) \text { for some } u \text { in } H_{1} \\
& =\Phi_{1}\left(u, S^{*}\left(S^{*}\right)^{-1} y\right) \\
& =\Phi_{1}(u, y) \\
& =\Phi_{1}\left(S^{-1} S u, y\right) \\
& =\Phi_{1}\left(S^{-1} x, y\right) .
\end{aligned}
$$

Therefore $\left(S^{-1}\right)^{*} \subseteq\left(S^{*}\right)^{-1}$ [2], [3]. Now let $R$ and $U$ be the mappings defined on $H_{1} \times H_{2} \rightarrow H_{2} \times H_{1}$ by

$$
\begin{aligned}
& R((x, y))=(y, x) \\
& U((x, y))=(-y, x) .
\end{aligned}
$$

Then, as can be easily checked, we have that $R, U$ are 1-1, onto; $R^{-1}\left(A^{\perp}\right)=R^{-1}(A)^{\perp}$ and $U\left(A^{\perp}\right)=U(A)^{\perp}$. Now

$$
\begin{aligned}
G\left(\left(S^{*}\right)^{-1}\right) & =\left\{\left(x,\left(S^{*}\right)^{-1} x\right): x \in H_{1}\right\} \\
& =\left\{\left(S^{*} u, u\right): u \in H_{2}\right\} \\
& =R^{-1}\left(G\left(S^{*}\right)\right) \\
& =R^{-1}\left(U\left(G(S)^{\perp}\right)\right) \\
& =R^{-1}(U(G(S)))^{\perp}
\end{aligned}
$$




$$
\begin{aligned}
G\left(\left(S^{-1}\right)^{*}\right) & =\left\{\left(S^{-1} y, y\right): y \in H_{2}\right\}^{\perp} \\
& =\left\{(-x, S x): x \in H_{1}\right\}^{\perp} \\
& =\left\{-R^{-1}(-S x, x): x \in H_{1}\right\}^{\perp} \\
& =\left\{-R^{-1} U(x, S x): x \in H_{1}\right\}^{\perp} \\
& =R^{-1}(U(G(S)))^{\perp} .
\end{aligned}
$$

Therefore the domain of $\left(S^{*}\right)^{-1}$ and that of $\left(S^{-1}\right)^{*}$ coincide. Hence [2], [3], we have that $\left(S^{-1}\right)^{*}=\left(S^{*}\right)^{-1}$.

Lemma 3.9. Let $\left(H_{i}, \Phi_{i}\right), i=1,2$ be quadratic spaces. If $S$ is a 1-1, onto orthocontinuous transformation on $H_{1}$ to $H_{2}$ then $S^{-1}$ is orthocontinuous.

Proof. By $3.8\left(S^{*}\right)^{-1}$ exists and equals $\left(S^{-1}\right)^{*}$. This shows that $S^{-1}$ is an everywhere defined linear transformation which is such that $\left(S^{-1}\right)^{*}$ everywhere defined. Therefore $S^{-1}$ is orthocontinuous [2], [3].

TheOREM 3.10. Let $\left(H_{i}, \Phi_{i}\right), i=1,2$ be quadratic spaces and $B$ an orthocontinuous bilinear form on $H_{1} \times H_{2}$. If $B_{x}(\cdot), B^{y}(\cdot)$ are nontrivial then there exists an orthocontinuous linear transformation, $T$, on $H_{2}$ to $H_{1}$ such that

$$
\Phi_{2}(u, v)=B(T u, v)
$$

for all $u, v$ in $H_{2}$. Further if $H_{2}$ is such that every $\perp$-closed subspace is splitting then $T$ is unique.

Proof. Let $L$ be the linear map on $H_{1}$ to $H_{2}$ associated with $B$ as in Definition 3.4. Then by 3.7, 3.8, and $3.9 T=L^{-1}$ is orthocontinuous and

$$
B(T u, v)=\Phi_{2}(u, v) \text { for all } u, v \text { in } H_{2} .
$$

Now suppose every $\perp$-closed subspace of $H_{2}$ splits $H_{2}$ Then the mapping $B_{x}: H_{2} \rightarrow K$ being orthocontinuous we have by [3, Theorem 3.1], that there exists a unique vector $u_{0}$ in $H_{2}$ such that $B_{x}(y)=$ $\Phi_{2}\left(u_{0}, y\right)$ for all $y$ in $H_{2}$. For each $x$ define $\widetilde{L} x=u_{0}$. Then $\widetilde{L}$ is linear and the uniqueness of $u_{0}$ implies that $\widetilde{L}=L$. Hence $L^{-1}=T$ is unique.

THEOREM 3.11. Let $\left(H_{i}, \Phi_{i}\right)$ be quadratic spaces and $B: H_{1} \times$ $H_{2} \rightarrow K$ an orthocontinuous bilinear form such that $B_{x}(\cdot), B^{y}(\cdot)$ are nontrivial. Then given any orthocontinuous linear functional $\phi$ on $H_{2}$ such that Ker $\phi$ splits $H_{2}$ there exists a unique vector $u_{0}$ in $H_{1}$ 
such that

$$
\phi(y)=B\left(u_{0}, y\right) \text { for all } y \text { in } H_{2} .
$$

Proof. Since Ker $\phi$ splits $H_{2}$ we have by [3, Theorem 3.1] that there exists a unique vector $y_{0}$ in $H_{2}$ such that $\phi(y)=\Phi_{2}\left(y_{0}, y\right)$ for all $y$ in $H_{2}$. Let $L$ be the orthocontinuous linear transformation associated with $B$ as in 3.4. Then by $3.7,3.8$, and $3.9 L^{-1}$ exists and is orthocontinuous. Put $u_{0}=L^{-1} y_{0}$. Then the uniqueness of $y_{0}$ and the fact that $L$ is a bijection implies that $y_{0}$ is unique. Also

$$
\begin{aligned}
\phi(y) & =\Phi_{2}\left(y_{0}, y\right) \\
& =\Phi_{2}\left(L L^{-1} y_{0}, y\right) \\
& =\Phi_{2}\left(L u_{0}, y\right) \\
& =B\left(u_{0}, y\right)
\end{aligned}
$$

for all $y$ in $H_{2}$.

In what follows we shall assume that the center of $K$ contains an ordered domain $P[1]$ and that

(i) for every $a$ in $K a a^{*}=|a|^{2}$,

(ii) for any $b$ in $P^{+}$there exists an element $c$ in $P^{+}$such that $c^{2}=b$. Put $c=b^{1 / 2}$.

Here $P^{+}$denotes the set of positive elements of $P .|a|$ is a defined in [2]. We also assume that $\sup _{v \neq 0}\left|\Phi_{2}(u, v)\right|\left(\left|\Phi_{2}(v, v)\right|^{1 / 2}\right)^{-1}$ exists for each $u$ in $H_{2}$ and that it is equal to $\left|\Phi_{2}(u, u)\right|^{1 / 2}$.

THEOREM 3.12. Let $\left(H_{i}, \Phi_{i}\right), i=1,2$ be quadratic spaces. With the above assumption on $K, \Phi_{1}, \Phi_{2}$ let $B$ be an orthocontinuous bilinear form satisfying:

3.12.1 $\sup _{x \in H_{1}} \mid B(x, y)$, exists and is in $P^{+}$for all $y \neq 0$.

3.12 .2

$$
\begin{gathered}
\inf _{x \neq 0} \sup _{y \neq 0}|B(x, y)|\left(\left|\Phi_{1}(x, y)\right|^{1 / 2}\left|\Phi_{2}(y, y)\right|^{1 / 2}\right)^{-1}=\gamma \\
\text { exists and } \gamma-\delta \text { is in } P^{+} \\
\text {for some } \delta \text { in } P^{+} .
\end{gathered}
$$

Then given any orthocontinuous linear functional $\phi$ on $H_{2}$ such that Ker $\phi$ splits $H_{2}$ there exists a unique element $x_{0}$ in $H_{1}$ such that

$$
\phi(y)=B\left(x_{0}, y\right) \text { for all } y \text { in } H_{2} .
$$

Moreover

$$
\delta^{-1} \sup _{y \neq 0}|\dot{\phi}(y)|\left(\left|\Phi_{2}(y, y)\right|^{1 / 2}\right)^{-1}-\left|\Phi_{1}\left(x_{0}, x_{0}\right)\right|^{1 / 2} \in P^{+} \cup\{0\} .
$$

Proof. By 3.12.1, 3.12.2 we have that $B_{x}(\cdot), B^{y}(\cdot)$ are non- 
trivial for each $x$ in $H_{1}$ and $y$ in $H_{2}$. Hence by 3.11 there exists a unique vector $x_{0}$ in $H_{1}$ such that $\phi(y)=B\left(x_{0}, y\right)$ for all $y$ in $H_{2}$. By condition 3.12 .2 we have

$$
\begin{aligned}
\left|\Phi_{1}\left(x_{0}, x_{0}\right)\right|^{1 / 2} & <\delta^{-1} \sup _{y \neq 0}\left|B\left(x_{0}, y\right)\right|\left(\left|\Phi_{2}(y, y)\right|^{1 / 2}\right)^{-1} \\
& =\delta^{-1} \sup _{y \neq 0}|\phi(y)|\left(\left|\Phi_{2}(y, y)\right|^{1 / 2}\right)^{-1} .
\end{aligned}
$$

REMARK 3. We note that a continuous linear functional on a pre-Hilbert space $H$, say, is orthocontinuous and if $H$ is a Hilbert space then the kernel of a continuous functional, which is a closed subspace, is splitting. Also if $H_{1}, H_{2}$ are pre-Hilbert spaces with $H_{2}$ complete then a continuous bilinear form on $H_{1} \times H_{2}$ is orthocontinuous. Hence the corollary that follows is an immediate consequence of the theorem.

Corollary 3.13. Let $H_{1}$ be a pre-Hilbert space and $H_{2}$ be a Hilbert space (both real, complex or quaternion) and $B(\cdot, \cdot)$ a bilinear form on $\mathrm{H}_{1} \times \mathrm{H}_{2}$ such that

$$
|B(x, y)| \leqq \delta^{\prime}\|x\|_{H_{1}}\|y\|_{H_{2}}
$$

for all $x$ in $H_{1}$ and $y$ in $H_{2}$ and for some positive real $\delta^{\prime}<\infty$,

$$
\begin{gathered}
\inf _{\|x\|_{H_{1}}=1} \sup _{\|y\|_{H_{2}} \leq 1}|B(x, y)| \geqq \delta>0, \\
\sup _{x \in H_{1}}|B(x, y)|>0 \text { for all } y \neq 0 .
\end{gathered}
$$

Let $\phi$ be a bounded linear functional on $H_{2}$. Then there exists a unique vector $x_{0}$ in $H_{1}$ such that

$$
\dot{\phi}(y)=B\left(x_{0}, y\right) \text { for all } y \text { in } H_{2} .
$$

Moreover

$$
\delta\left\|x_{0}\right\|_{H_{1}}=\|\phi\|
$$

REMARK 4. We note from the foregoing that the completeness of $H_{1}$ is not necessary for these results to hold unless $H_{1}=H_{2}$; in which case we obtain the Lax-Milgram Theorem.

\section{REFERENCES}

1. B. Birkhoff and S. MacLane, A Survey of Modern Algebra, Macmillan Company, New York, 1965.

2. R. Piziak, An Algebraic Generalization of Hilbert Space Geometry, Ph. D. Thesis, Univ. of Mass., 1969. 
3. H. Piziak, Sesquilinear forms in infinite dimensions, Pacific J. Math., 43 (1972), 475-481.

4. K. Yosida, Functional Analysis, Springer-Verlag, Berlin, Heidelberg, New York, 1971.

Received September 8, 1978 and in revised form May 1, 1979.

UNIVERSITY OF BENIN

Benin-City, Nigeria 


\title{
PACIFIC JOURNAL OF MATHEMATICS
}

\section{EDITORS}

\author{
DoNALD BABBITT (Managing Editor) \\ University of California \\ Los Angeles, CA 90024 \\ HUGo RossI \\ University of Utah \\ Salt Lake City, UT 84112 \\ C. C. MOORE and ANDREW OGG \\ University of California \\ Berkeley, CA 94720
}

J. DugundJI

Department of Mathematics

University of Southern California

Los Angeles, CA 90007

R. FINN and J. Milgram

Stanford University

Stanford, CA 94305

\section{ASSOCIATE EDITORS}
E. F. BeCKenbach
B. H. NeumanN
F. WOLF
K. YoshidA

\section{SUPPORTING INSTITUTIONS}

\author{
UNIVERSITY OF BRITISH COLUMBIA \\ CALIFORNIA INSTITUTE OF TECHNOLOGY \\ UNIVERSITY OF CALIFORNIA \\ MONTANA STATE UNIVERSITY \\ UNIVERSITY OF NEVADA, RENO \\ NEW MEXICO STATE UNIVERSITY \\ OREGON STATE UNIVERSITY \\ UNIVERSITY OF OREGON
}

\author{
UNIVERSITY OF SOUTHERN CALIFORNIA \\ STANFORD UNIVERSITY \\ UNIVERSITY OF HAWAII \\ UNIVERSITY OF TOKYO \\ UNIVERSITY OF UTAH \\ WASHINGTON STATE UNIVERSITY \\ UNIVERSITY OF WASHINGTON
}

The Supporting Institutions listed above contribute to the cost of publication of this Journal, but they are not owners or publishers and have no responsibility for its content or policies.

Mathematical papers intended for publication in the Pacific Journal of Mathematics should be in typed form or offset-reproduced, (not dittoed), double spaced with large margins. Please do not use built up fractions in the text of the manuscript. However, you may use them in the displayed equations. Underline Greek letters in red, German in green, and script in blue. The first paragraph or two must be capable of being used separately as a synopsis of the entire paper. Please propose a heading for the odd numbered pages of less than 35 characters. Manuscripts, in triplicate, may be sent to any one of the editors. Please classify according to the scheme of Math. Reviews, Index to Vol. 39. Supply name and address of author to whom proofs should be sent. All other communications should be addressed to the managing editor, or Elaine Barth, University of California, Los Angeles, California, 90024.

50 reprints to each author are provided free for each article, only if page charges have been substantially paid. Additional copies may be obtained at cost in multiples of 50 .

The Pacific Journal of Mathematics is issued monthly as of January 1966. Regular subscription rate: $\$ 84.00$ a year $(6$ Vols., 12 issues). Special rato: $\$ 42.00$ a year to individual members of supporting institutions.

Subscriptions, orders for numbers issued in the last three calendar years, and changes of address shoud be sent to Pacific Journal of Mathematics, P.O. Box 969, Carmel Valley, CA 93924, U.S.A Old back numbers obtainable from Kraus Periodicals Co., Route 100, Millwood, NY 10546.

\section{PUBLISHED BY PACIFIC JOURNAL OF MATHEMATICS, A NON-PROFIT CORPORATION}

Printed at Kokusai Bunken Insatsusha (International Academic Printing Co., Ltd.). 8-8, 3-chome, Takadanobaba, Shinjuku-ku, Tokyo 160, Japan. 


\section{Pacific Journal of Mathematics}

\section{Vol. 86, No. 2 December, 1980}

Graham Donald Allen, David Alan Legg and Joseph Dinneen Ward, Hermitian

liftings in Orlicz sequence spaces ............................... 379

George Bachman and Alan Sultan, On regular extensions of measures ........ 389

Bruce Alan Barnes, Representations Naimark-related to $*$-representations; a

correction: "When is a representation of a Banach $*$-algebra

Naimark-related to a $*$-representation?" ........................ 397

Earl Robert Berkson, One-parameter semigroups of isometries into $H^{p} \ldots \ldots .403$

M. Brodmann, Piecewise catenarian and going between rings ............ 415

Joe Peter Buhler, A note on tamely ramified polynomials ............... 421

William Lee Bynum, Normal structure coefficients for Banach spaces ........ 427

Lung O. Chung, Biharmonic and polyharmonic principal functions ......... 437

Vladimir Drobot and S. McDonald, Approximation properties of polynomials

with bounded integer coefficients .............................

Giora Dula and Elyahu Katz, Recursion formulas for the homology of

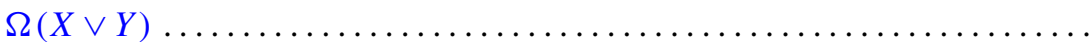

John A. Ernest, The computation of the generalized spectrum of certain Toeplitz operators ...................................... 463

Kenneth R. Goodearl and Thomas Benny Rushing, Direct limit groups and the

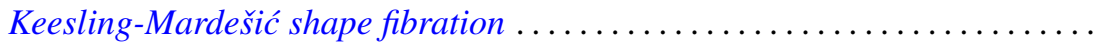

Raymond Heitmann and Stephen Joseph McAdam, Good chains with bad contractions

Patricia Jones and Steve Chong Hong Ligh, Finite hereditary near-ring-semigroups .

Yoshikazu Katayama, Isomorphisms of the Fourier algebras in crossed

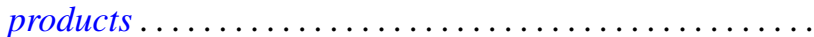

Meir Katchalski and Andrew Chiang-Fung Liu, Symmetric twins and common transversals. .

Mohammad Ahmad Khan, Chain conditions on subgroups of LCA groups ....

Helmut Kröger, Padé approximants on Banach space operator equations ...

Gabriel Michael Miller Obi, An algebraic extension of the Lax-Milgram theorem . .

S. G. Pandit, Differential systems with impulsive perturbation .

Richard Pell, Support point functions and the Loewner variation ...

J. Hyam Rubinstein, Dehn's lemma and handle decompositions of some

4-manifolds

James Eugene Shirey, On the theorem of Helley concerning finite-dimensional subspaces of a dual space.

Oved Shisha, Tchebycheff systems and best partial bases.... 